\title{
The effect of fungal iron uptake on olivine weathering studied by genetic approaches in the rock-inhabiting fungus Knufia petricola
}

\author{
RUBEN GERRITS ${ }^{1}$, MICHAEL J HENEHAN ${ }^{2}$, INES \\ FELDMANN $^{3}$, JÖRG RADNIK ${ }^{3}$, LEONARDO AGUDO \\ JÁCOME $^{3}$, JAN LISEC ${ }^{3}$, JACQUES SCHOTT ${ }^{4}$, JULIA \\ SCHUMACHER $^{3,5}$, ANNA A. GORBUSHINA ${ }^{3,5}$ AND \\ FRIEDHELM VON BLANCKENBURG ${ }^{2}$
}

${ }^{1}$ Bundesanstalt für Materialforschung und -Prüfung

${ }^{2}$ GFZ German Research Centre for Geosciences

${ }^{3}$ Bundesanstalt für Materialforschung und Prüfung

${ }^{4}$ GET-OMP-CNRS-Toulouse University

${ }^{5}$ Freie Universität Berlin

Presenting Author: ruben.gerrits@bam.de

Fungi are known to accelerate silicate weathering and therefore, not only affect soil formation, but are also relevant to terrestrial enhanced weathering (EW). Knowledge of the mechanisms by which fungi accelerate weathering is vital if we are to exploit their EW potential. By deleting certain genes in fungi, their individual roles in weathering reactions can be disclosed. Our previous research has indicated that (1) iron oxidation at the surface of olivine $\left(\mathrm{Mg}_{1.8} \mathrm{Fe}_{0.2} \mathrm{SiO}_{4}\right)$ inhibits its dissolution and (2) attachment of the rock-inhabiting fungus Knufia petricola is able to prevent this inhibition through iron sequestration. Here we explored whether these effects are related to iron uptake by $K$. petricola. Genes encoding a putative siderophore synthetase and a ferric reductase were deleted. These mutants or the wild type (WT) of $K$. petricola were incubated in flow-through dissolution experiments buffered at $\mathrm{pH} 4$ or 6 , running an abiotic control in parallel. Olivine dissolution kinetics was quantified based on bulk fluid chemical analyses. Aqueous iron isotopic fractionation was studied using MC-ICP-MS, siderophore production using HPLC-MS and olivine's surface chemistry and structure using XPS and FIB-TEM, respectively.

Preliminary results show that the WT and the mutants sequestered equal amounts of iron and dissolved olivine at a similar rate. Interestingly, the WT and mutants enhanced olivine dissolution compared to the abiotic control at $\mathrm{pH} 6$ but not at $\mathrm{pH}$ 4. Furthermore, the concentration and isotopic fractionation of dissolved iron suggest that olivine-released iron stayed in

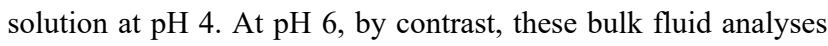
imply that iron precipitated under abiotic conditions but was kept in solution in the presence of fungi. However, using XPS, no iron oxide precipitation was detected on the olivine surface from the abiotic control at $\mathrm{pH}$ 6. Altogether, these results show that (1) a different set of deletion mutants is needed to study the effect of fungal iron uptake and (2) the slow iron oxidation kinetics at $\mathrm{pH}$ 4 prevents $K$. petricola from enhancing dissolution. The latter indicates the importance of fungal iron sequestration in silicate dissolution and the accelerating effect of fungi on $\mathrm{EW}$ in $\mathrm{pH}$ neutral soils. 